\title{
A New Biomechanical Model Based Approach on Brain Shift Compensation
}

\author{
Keiji Kobashi $^{1,2}$, Xenophon Papademetris ${ }^{2}$, and James S. Duncan ${ }^{2,3}$ \\ 1 Power and Industrial Systems R\&D Lab., Hitachi Ltd., Ibaraki, Japan, \\ 2 Dept. of Diag. Radiology, keiji_kobashi@pis.hitachi.co.jp \\ 3 Electrical Engineering, Yale University, CT, USA
}

\begin{abstract}
We propose a new algorithm for biomechanical model-based brain shift compensation in image guided neurosurgery. It can be used to update preoperative images with intraoperatively acquired information. We derive a model equation with regard to external forces acting on the brain surface during neurosurgery which can be consistently integrated with intraopearatively acquired information, assuming that these forces induce a linear biomechanical response. We treat external forces on the brain boundaries as unknown variables and then estimate them within a framework of inverse finite element analysis. By incorporating additional constraints from prior knowledge, we can solve the derived equations to obtain reasonable estimation results on boundary forces and the entire displacement field. This algorithm is especially beneficial in reducing navigation error of deeper brain structures by updating preoperative images using only exposed surface displacement. In this paper, we describe the derivation of the equations and present examples of two dimensional synthetic data, where the estimated displacement errors are reduced by fifty percent, compared to the standard approach.
\end{abstract}

\section{Introduction}

Correction of navigation error due to intraoperative brain shift has become a central issue in the context of improving accuracy of image guided neurosurgery(IGNS). According to published measurements $[2,6]$, brain surface displacement during neurosurgery can be as much as $10 \mathrm{~mm}$ or larger, and thus brain shift may be the dominant cause of navigation error. To cope with this problem, computational image correction is often employed, in which preoperative images are updated using intraoperatively acquired information. Ferrant et al. use intraoperatively acquired volumetric MR image sequences [1], however, most other groups use displacement estimates of open brain surfaces $[4,8]$ for brain shift compensation. In these latter cases, a shortage of information on boundary condition makes image registration ill-posed, and thus additional a priori information is required for better estimation of entire displacement of brains.

Since we treat non-rigid brain deformation, non-rigid image registration is necessary. Recently, a biomechanical model based approach has been increasingly 
employed for this purpose since it can incorporate real physics, such as material inhomogeneity, anisotropy, and so on. Finite element modeling may be the most viable way to deal with such problems.

With regard to biomechanical modeling of brains, Kyriacou et al's recent investigation is useful [3]. They state that the choice of the appropriate material model depends on the application, and characteristic time scale of the phenomenon is a very important factor. For example, a viscoelastic solid model may be the most appropriate for quasi-static processes, if there is no specific requirement for knowing the interstitial fluid movement. However, they also suggest that a linear elastic model may be enough for image registration.

While investigation of material properties has been a central topic in the study of brain deformation for a long time, little has been published on boundary and external loading conditions. It is no doubt that gravity is the originating factor of the so called "sinking" deformation $[2,6]$, however, complications arise when dealing with its influence, because of its highly complex in-vivo characteristics such as loss of cerebro-spinal fluid(CSF), buoyancy change, brain-skull interaction and so on. Without intraoperative 3D imaging, as in Ferrant et al [1], we can only access displacement of open brain surfaces, which is not sufficient to estimate deeper structures' displacement. To deal with such problems, challenging approaches have been conducted by Miga et al. who modeled buoyancy change due to the loss of CSF [4], and by Skrinjar et al. who incorporated brain-skull contact model into their analysis [7]. Both attempts have included the interaction between the brain and its surroundings into their biomechanical models. On the other hand, our method aims to identify the influences of those interactions using intraoperatively observed open brain surface displacement.

Brain sinking after opening the dura is the most significant source of deformation during neurosurgery. A rough sketch of the mechanism of brain sinking is as follows: opening the arachnoid membrane causes a pressure leak and loss of CSF, this loss of CSF causes a hydrostatic pressure change (part of brain surfaces are subject to air pressure), then the brain sinks and contact with the skull and surrounding structures results in reaction forces that push back the brain through contact surface and support the increased brain weight. But if we see this process from inside the brain, all the phenomena are just actions of external forces whatever may be the cause. From this perspective, the focus is on the external forces themselves.

In this paper, we derive a key equation in terms of external forces, which can account for intraoperatively observed information on brain shift. Then we introduce some additional constraints, incorporating prior knowledge on external force distributions, providing us with unique solutions. We test our algorithm using synthetic 2D data.

\section{Methods}

\subsection{Definition of Problem}

Here we define the biomechanical system as is depicted in figure 1. We divide the entire object into four regions. Region 1 is a region where displace- 
ment is fixed (or may be prescribed) through prior knowledge. Reaction forces are obtained as a biomechanical response to inferred external forces on region 2 and 3. Region 2 is a region where displacement is observed. This observed displacement is used as input data, or as landmarks, for registration. External forces acting on this region are unknowns to be estimated. This region (2), corresponds to open brain surfaces in neurosurgery. Region 3 is a region where external forces are to be estimated and the displacements are obtained as a solution. It corresponds to hidden brain surfaces in neurosurgery. Region 4 is a region where displacement is to be obtained as a biomechanical response. Body forces can be distributed, but here we treat them as zero for simplicity. It corresponds to the interior regions of the brain.

Our algorithm aims at identifying external forces acting on the boundaries, specifically region 2 and 3 . Note that once external forces on region 2 and 3 are determined, all other unknowns can be obtained by standard straightforward analyses.

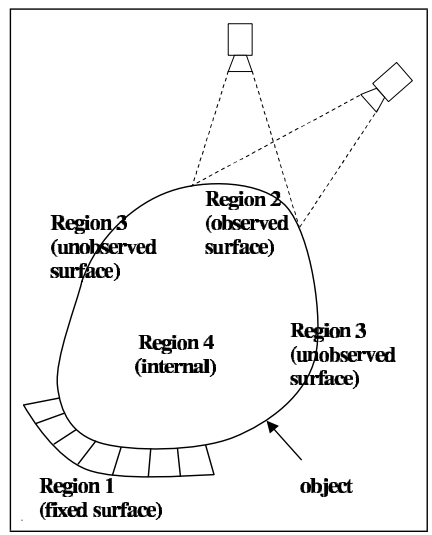

Fig. 1. Definition of the Biomechanical System

\subsection{Derivation of Fundamental Equation}

A linearized, discretized version of the equilibrium equation for brain deformation between two image sequences, can be written as

$$
\left[\begin{array}{llll}
k_{11} & k_{12} & k_{13} & k_{14} \\
k_{21} & k_{22} & k_{23} & k_{24} \\
k_{31} & k_{32} & k_{33} & k_{34} \\
k_{41} & k_{42} & k_{43} & k_{44}
\end{array}\right]\left\{\begin{array}{l}
u_{1}^{*} \\
u_{2}^{*} \\
u_{3} \\
u_{4}
\end{array}\right\}=\left\{\begin{array}{l}
f_{1} \\
f_{2} \\
f_{3} \\
f_{4}^{*}
\end{array}\right\},
$$

where $u_{i}$ is a displacement vector of region $i, f_{i}$ is an external force vector of region $i, k_{i j}$ is a sub matrix of the global stiffness matrix relating to region $i$ and $j$. The $i$ index (row) refers to $i$-th weighted residual equation, and the $j$ index (column) to the contribution of the $j$-th coefficient associated with a basic function expansion. Upper suffix * means that the variable is "known" (omitted from $k_{i j}$, for simplicity). $u_{1}^{*}$ is known from the definition. Typically we give $u_{1}^{*}=0 . f_{4}^{*}$ is also known from the definition, typically $f_{4}^{*}=0 . u_{2}^{*}$ is given by observation. You might think $f_{2}$ should be zero as the region 2 is exposed to air after opening the dura. But it is not zero at least for the first step of image correction because $f_{2}$ means force difference between image sequences and there is always non-zero force on the surface until opening the dura. Conversely, when we apply this algorithm using two image sequences both after opening the dura to follow brain shift, the assumption of $f_{2}=0$ would be beneficial. 
In standard straightforward finite element analyses, it is required that we should give either $u_{i}$ or $f_{i}$ as known in each row, making equilibrium equations regular. But in above Eqn.(1), we have two unknowns, $u_{3}$ and $f_{3}$ in a third row, meaning that this equation is under-determined and must be treated in a different manner from standard finite element analysis.

Let us consider identifying $f_{2}$ and $f_{3}$. We use a similar manner to condensation procedures in FEM. At first, suppose we are given $f_{2}, f_{3}$ and solve for $u_{2}$ in Eqn.(11). We would have $u_{2}, u_{3}, u_{4}$ and $f_{1}$ as a unique solution, but $u_{2}$ obtained this way would not be the observed $u_{2}^{*}$. The aim of this setup is to find the $f_{2}$ and $f_{3}$ which make $u_{2}$ correspond to the observed $u_{2}^{*}$.

From the second row of Eqn.(1), we obtain

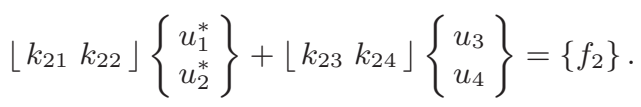

From the third and fourth row of Eqn. (1), we have

$$
\left[\begin{array}{ll}
k_{33} & k_{34} \\
k_{43} & k_{44}
\end{array}\right]\left\{\begin{array}{l}
u_{3} \\
u_{4}
\end{array}\right\}=\left\{\begin{array}{l}
f_{3} \\
f_{4}^{*}
\end{array}\right\}-\left[\begin{array}{ll}
k_{31} & k_{32} \\
k_{41} & k_{42}
\end{array}\right]\left\{\begin{array}{l}
u_{1}^{*} \\
u_{2}^{*}
\end{array}\right\} .
$$

Since the left side coefficient matrix is regular, it has a unique inverse matrix(currently the calculation cost may be problematic for large scale problems), which can be written as:

$$
\left[\begin{array}{ll}
k_{33} & k_{34} \\
k_{43} & k_{44}
\end{array}\right]^{-1}=[H]=\left[\begin{array}{ll}
h_{33} & h_{34} \\
h_{43} & h_{44}
\end{array}\right]
$$

Then we obtain

$$
\left\{\begin{array}{l}
u_{3} \\
u_{4}
\end{array}\right\}=[H]\left[\left\{\begin{array}{l}
f_{3} \\
f_{4}^{*}
\end{array}\right\}-\left[\begin{array}{ll}
k_{31} & k_{32} \\
k_{41} & k_{42}
\end{array}\right]\left\{\begin{array}{l}
u_{1}^{*} \\
u_{2}^{*}
\end{array}\right\}\right] .
$$

Substituting this into Eqn.(2) and eliminating $u_{3}$ and $u_{4}$, we have

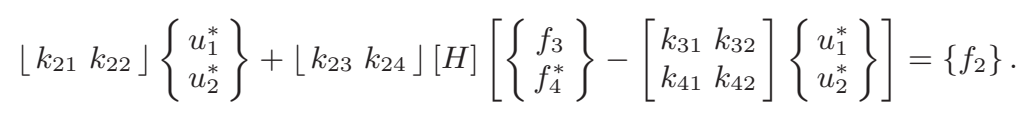

Further substitutions and setting $u_{1}^{*}=0$ and $f_{4}^{*}=0$, results in

$$
[P, Q]\left\{\begin{array}{l}
f_{2} \\
f_{3}
\end{array}\right\}=\left\{u_{2}^{*}\right\}
$$

where $P=R_{22}^{-1}, Q=R_{22}^{-1} X_{23}, X_{23}=-\left(k_{23} h_{33}+k_{24} h_{43}\right)$ and

$$
R_{22}=k_{22}-\left\lfloor k_{23} k_{24}\right\rfloor\left[\begin{array}{ll}
h_{33} & h_{34} \\
h_{43} & h_{44}
\end{array}\right]\left[\begin{array}{l}
k_{32} \\
k_{42}
\end{array}\right] .
$$

When we substitute: $A=[P, Q], x^{t}=\left\lfloor f_{2}, f_{3}\right\rfloor$ and $b=u_{2}^{*}$, Eqn.(7) can be expressed in a simple simultaneous equation form.

$$
A x=b
$$


This equation is under-determined, but it gives a significant restriction on external force distributions. If an external force vector $x$ satisfies this equation, and is used to infer the entire displacement field, then the obtained $u_{2}$ will correspond to the observed brain surface displacement $u_{2}^{*}$. The derivation of this equation may appear tricky, but it can be interpreted as a practical application of Maxwell-Betti's reciprocal theorem in mechanics.

Solving the Equation: Additional constraints are required to solve Eqn.(8). We employed a mathematical regularization scheme in this preliminary work. Using singular value decomposition(SVD), we can obtain a generalized inverse matrix of a non-square matrix uniquely. Applying this technique to the matrix $A$ in Eqn.(8), we can obtain a solution. It is a minimum norm solution in terms of $x$, reflecting our prior knowledge that the force vector would not be so large.

\subsection{Weighting of Force Terms}

Brain shift after dura opening is one of the most drastic changes during neurosurgery. It is thought that this shift happens mainly due to the leakage of CSF, causing pressure change over the entire brain surfaces. In other words, what mainly causes brain deformation is not the external forces acting on exposed brain surfaces but forces acting on unexposed brain surfaces as well. To incorporate this physical insight into analyses, we adopted a parameter to control the relative contribution of $f_{2}$ and $f_{3}$. Expanding Eqn.(7), we have:

$$
P f_{2}+Q f_{3}=u_{2}^{*} \text {. }
$$

We note that $u_{2}^{*}$ is expressed as a linear combination of two terms, $f_{2}$ and $f_{3}$ respectively. We can re-express the above equations with the appropriate substitutions as:

$$
\begin{gathered}
\alpha P f_{2}^{\prime}+(1-\alpha) Q f_{3}^{\prime}=u_{2}^{*}, \\
\left\{\begin{array}{l}
f_{2} \\
f_{3}
\end{array}\right\}=\left[\begin{array}{ll}
\alpha & 0 \\
0 & (1-\alpha)
\end{array}\right]\left\{\begin{array}{l}
f_{2}^{\prime} \\
f_{3}^{\prime}
\end{array}\right\}=L x^{\prime}(=x) .
\end{gathered}
$$

Introducing matrix $W$, Eqn.(10) is expressed as

$$
W x^{\prime}=b,
$$

where

$$
W(\alpha)=[\alpha P,(1-\alpha) Q], \quad(0 \leq \alpha \leq 1) .
$$

Solving this matrix equation in terms of $x^{\prime}$ again using SVD, $\alpha$ acts as a regional weighting factor which determines the contribution of $f_{2}$ and $f_{3}$. When $\alpha=1$, only $f_{2}$ is used. This is equivalent to the conventional method giving a prescribed displacement at exposed brain surfaces, often adopted in biomechanical model based non-rigid registration [5,8]. On the contrary, when $\alpha=0$, only $f_{3}$ is used and $f_{2}$ becomes zero. If we set $\alpha=1 / 2$, it is equivalent to Eqn.(17), which tends to use $f_{2}$ than $f_{3}$ because of the geometrical distance between region 2 and 3. To increase estimation accuracy of deeper brain regions, we need to increase the contribution of $f_{3}$, although it strongly depends on the case and must be determined empirically. Systematic investigation using intra-operative data will be indispensable on this issue. 


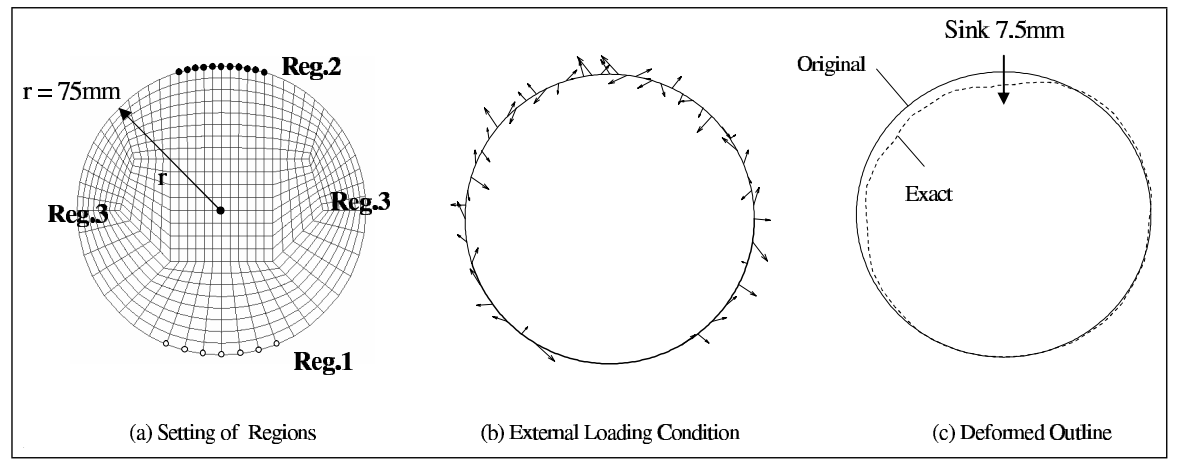

Fig. 2. A homogeneous, isotropic solid circle $(\mathrm{r}=75 \mathrm{~mm})$. Poisson's ratio is set to 0.45 . (a) Setting of Regions, (b) External loading condition. Forces are distributed on each node randomly both in magnitude and direction. A random force distribution in which the top surface sinks is selected for testing. (c) Outline of deformed shape obtained from straightforward finite element analysis under the loading condition of (b). The magnitude of external load is adjusted so that the maximum displacement in the sinking direction becomes $7.5 \mathrm{~mm}$.

\section{Experiments}

We have tested our algorithm on a synthetic two dimensional problem shown in figure 2. The bottom side is fixed(supposed as a brain stem or other rigid part of a brain), and the displacement is to be observed at the top side(supposed as an open brain surface). Figure 2(b) presents the external loading condition which gives deformation depicted in figure 2(c), conceptually mimicking the mechanism of brain sinking after dura opening. We added randomness to the external loading so that the test problem would not be too intentional. Here we assumed that brain sinking is quite related with external forces on unobserved, hidden boundary surfaces, mainly because the area of the hidden surfaces are usually much larger that of the open brain surfaces. If this assumption is inappropriate, the conventional prescribed displacement method, which is usually used for biomechanical model based non-rigid image registration [5], would give us satisfying solutions. Conversely, if the assumption is appropriate, we would need to incorporate the effect of external forces on unobserved surfaces. It cannot be achieved in conventional methods. Considering that the open part of the brain surface is much smaller than the hidden part of the surface in most cases, our assumption would be appropriate. From this physical insight, we have introduced the regional weighting method and provided the loading condition depicted in figure 2(b). We then estimate the entire displacement field using only the top side displacement and the knowledge of bottom side fixation. We subdivide the entire region as is shown in figure 2(a) for finite element discretization. The outlines from the estimation results are depicted in figure 3. We have tested three different algorithms including (a)conventional prescribed displacement method using straightforward finite element analysis, (b)our algorithm without weighting using Eqn. (8), and (c)our algorithm with weighting using Eqn.12). We set 


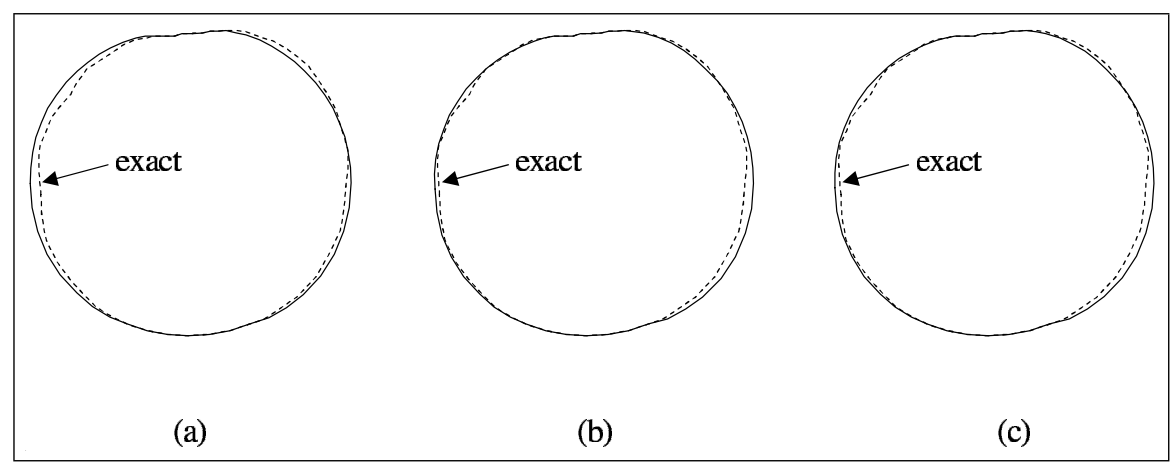

Fig. 3. Analysis Results. Outlines obtained from each method for the test problem depicted in figure 2. Dashed lines show the exact solution. (a) Conventional FEA with top side prescribed, (b) unweighted(Eqn.8), and (c) weighted(Eqn.12, a=0.55). Deformation in horizontal direction is better recovered in the proposed methods (b) and (c) than in the conventional method (a).

\begin{tabular}{|c|r|r|r|r|}
\hline \multicolumn{9}{|c|}{ Absolute Value[mm] } & \multicolumn{2}{c|}{ Ratio } \\
\hline & Shallow & Deep & Shallow & Deep \\
\hline Conventional & 0.693 & 0.889 & 1.000 & 1.000 \\
\hline Unweighted (Eqn.10) & 0.318 & 0.508 & 0.459 & 0.571 \\
\hline Weighted (Eqn.14) & 0.316 & 0.436 & 0.456 & 0.490 \\
\hline \multicolumn{7}{|c|}{} \\
\hline
\end{tabular}

Fig. 4. Estimation Accuracy. Root mean square of errors integrated in the shallow and deep areas are shown in the table. With our method, the RMS errors are reduced by 50 percent in both in the shallow and the deep areas.

$\alpha=0.55$ in this case so that the displacement error norm is minimized through parameter search. The exact solution is shown with a dashed line in each figure. In figure 3(a), the top side displacement is completely satisfied but there are considerable errors in the horizontal direction. The error arises from the assumption that there is no loading on the left and right sides. On the other hand, in figure 2(b) and (c), errors in horizontal direction are reduced, especially the area close to the top side. The external loads on the left and right sides helped to recover the horizontal deformation. Error norms are compared in figure 4 . Root mean square errors (RMS) of displacement estimation on the nodes are calculated in both shallow and deep areas. In the shallow area, RMS is reduced from (a) $0.693 \mathrm{~mm}$ to (b) $0.318 \mathrm{~mm}$ and (c) $0.316 \mathrm{~mm}$, decreasing by 45 percent. In the deep area, where errors are larger than the shallow area, RMS is reduced from (a) $0.889 \mathrm{~mm}$ to (b) $0.508 \mathrm{~mm}$ and (c) $0.436 \mathrm{~mm}$, decreasing by 57 and 49 percent respectively. These results indicate that displacement estimation errors can be reduced by 50 percent using our methods. The weighted version is especially beneficial in reducing errors of deeper areas. We note that the parameter $\alpha$ must be chosen appropriately. 


\section{Conclusions}

We have proposed a new biomechanical model based non-rigid registration algorithm within the framework of finite element inverse analysis for the purpose of improving accuracy of IGNS. It can be used to update preoperative images with intraoperatively acquired information. Assuming that the brain deformation is a linear elastic response to external forces, we have derived an essential equation describing the relationship between unknown external forces acting on boundaries and observed displacement. By solving the equation with some additional constraints from a priori information, we can estimate unknown external forces reasonably. Then the entire displacement field can be obtained as a response to the estimated external forces. From two dimensional synthetic data mimicking brain sinking, we have tested the validity and efficacy of our algorithm. These preliminary results suggest that navigation errors can be reduced by fifty percent using this method. Future work will involve validation of this methodology using intra-operative data.

Acknowledgments. This work has been done during Kobashi's stay as a visiting research scientist at Yale University. We would like to thank PIS Lab., Hitachi Ltd. for giving us the opportunity to perform this research.

\section{References}

[1] M. Ferrant, A. Nabavi, B. Macq, F.A. Jolesz, R. Kikinis, and S.K. Warfield. Registration of 3D Intraoperative MR Images of the Brain Using a Finite Element Biomechanical Model. IEEE Transactions on Medical Imaging, 20(12): 1384-1397, December 2001.

[2] D.L.G. Hill, C.R.J. Maurer, R.J.Maciunas, J.A. Barwise, M.J. Fitzpatrick, and M.Y. Wang. Measurement of Intraoperative Brain Surface Deformation under a Craniotomy. Neurosurgery, 43(3): 514-526, September 1998.

[3] S.K. Kyriacou, A. Mohamed, K. Miller, and S. Neff. Brain Mechanics for Neurosurgery: Modeling Issues. Biomechanics and Modeling Mechanobiology, 1(2): 151$164,2002$.

[4] M.I. Miga, K.D. Paulsen, J.M. Lemery, S.D. Eisner, A. Hartov, F.E. Kennedy, and D.W. Roberts. Model-Updated Image Guidance: Initial Clinical Experience with Gravity-Induced Brain Deformation. IEEE Transactions on Medical Imaging, 18(10), 866-874, October 1999.

[5] W. Peckar, C.S. Karl Rohr, and H.S. Stiehl. Parameter-Free Elastic Deformation Approach for 2D and 3D Registration Using Prescribed Displacements Journal of Mathematical Imaging and Vision, 10, 143-162, 1999

[6] D.W. Roberts, A. Hartov, F.E. Kennedy, M.I. Miga, and K.D. Paulsen. Intraoperative Brian Shift and Deformation: A Quantitative Analysis of Cortical Displacement in 28 Cases. Neurosurgery, 43(4): 749-758, October 1998.

[7] O. Skrinjar, D. Spencer and J. Duncan. Brain Shift Modeling for Use in Neurosurgery, Medical Image Computing and Computer-Assisted Intervention (MICCAI 98), Cambridge, MA, USA, 641-649, October 1998.

[8] O. Skrinjar, A. Nabavi, and J. Duncan. Model-driven Brain Shift Compensation, Medical Image Analysis, 6: 361-373, 2002. 